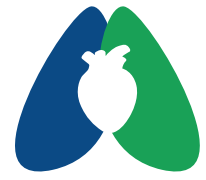

ASSOBRAFIR

C I Ê N C I A

\title{
Perfil dos pacientes submetidos à fisioterapia em uma Unidade de Terapia Intensiva do Extremo Sul da Bahia, Brasil
}

\author{
Profile of patients undergoing physiotherapy in an Intensive Care \\ Unit in the Extreme South of Bahia, Brazil
}

\author{
Sanielle Freire Reis ${ }^{1 *}$ (1); Henika Priscila Lima Silva² (1); Marília Caixeta de Araújo $^{3}$ (1); \\ Ariane Dórea Santos ${ }^{4}$ \\ ${ }^{1}$ Faculdades Integradas do Extremo Sul da Bahia, Eunápolis, BA, Brasil \\ ${ }^{2}$ Programa de Pós-graduação em Estado e Sociedade, Universidade Federal do Sul da Bahia (UFSB), Porto \\ Seguro, BA, Brasil \\ ${ }^{3}$ Especialização em Residência Integrada Multiprofissional em Saúde Cardiovascular Fisioterapia, \\ Universidade Federal de Minas Gerais (UFMG), Belo Horizonte, MG, Brasil \\ ${ }^{4}$ Associação Brasileira de Fisioterapia Cardiorrespiratória e Fisioterapia em Terapia Intensiva (ASSOBRAFIR) \\ São Paulo, SP, Brasil
}

Como citar: Reis SF, Silva HPL, Araújo MC, Santos AD. Perfil dos pacientes submetidos à fisioterapia em uma Unidade de Terapia Intensiva do Extremo Sul da Bahia, Brasil. ASSOBRAFIR Ciênc. 2021;12:e41995. https:// doi.org/10.47066/2177-9333.AC.2020.0027

\begin{abstract}
Resumo
Introdução: Caracterizada por serviços de alta complexidade, a Unidade de Terapia Intensiva (UTI), recebe pacientes com diferentes níveis de gravidade que necessitem de atendimento continuo, imediato e complexo. A coleta de dados sociodemográficos e clínicos acerca desses pacientes possibilita a analise populacional, que é fundamental para planejamento dos serviços de saúde, em todos os níveis de atenção. Objetivo: Traçar o perfil dos pacientes atendidos na Unidade de Terapia Intensiva adulto do Hospital Regional de Eunápolis, Bahia. Método: Foi realizado um estudo transversal, documental, retrospectivo, de caráter exploratório e abordagem descritiva, através da análise de prontuários de pacientes admitidos e atendidos pela fisioterapia na Unidade de Terapia Intensiva do Hospital Regional de Eunápolis - BA, no período de 01 de março de 2019 à 31 de março de 2020. Resultados: Foram analisados 321 prontuários, onde o perfil predominante foi de indivíduos do sexo masculino (54,2\%), com idade média de 58,64 anos e tempo de internação inferior a uma semana. As alterações cardiovasculares $(39,9 \%)$ e do sistema respiratório $(19,3 \%)$ foram os principais motivos de internação. As comorbidades associadas estavam ausentes em $57,9 \%$ dos pacientes. Em relação ao desfecho da internação 55,45\% tiveram alta hospitalar, 36,45\% óbito e $8,10 \%$ transferência; $52 \%$ dos pacientes necessitaram de ventilação mecânica no decorrer da internação. Conclusão: Este estudo reconheceu o perfil do público atendido em uma UTI de homens com meia idade, acometidos por eventos cardiovasculares, servindo de subsídio para a adoção de práticas em saúde de prevenção, promoção e reabilitação específicas ao público identificado.
\end{abstract}

Palavras-chave: Perfil de Saúde; Unidade de Terapia Intensiva; Fisioterapia.
Submissão em: Outubro 29, 2020

Aceito em: Agosto 09, 2021

Estudo realizado em: Faculdades integradas do Extremo Sul da Bahia, Eunápolis, BA, Brasil.

Aprovação ética: CAEE

30003520.5.0000.5526 da Universidade

Estadual de Santa Cruz, n 3.975 .088

*Autor correspondente:

Sanielle Freire Reis. E-mail:

saniellereisfisioterapia@gmail.com

\section{Abstract}

Background: Characterized by highly complex services, the Intensive Care Unit (ICU) receives patients with different levels of severity who need continuous, immediate and complex care. The collection of sociodemographic and clinical data about these patients enables population analysis, which is essential for planning health services at all levels of care. Aim: To trace the profile of patients seen at the adult Intensive Care Unit of the Regional Hospital of Eunápolis, Bahia. Methods: A cross-sectional, documental, retrospective, exploratory study with a descriptive approach was conducted through the analysis of medical records of patients admitted and treated by physical therapy in the Intensive Care Unit of the Hospital Regional de Eunápolis - BA, in the period of March 1st from 2019 to March 31, 2020. Results: A total of 321 medical records were analyzed, where the predominant profile was male (54.2\%), with a mean age of 58.64 years and hospitalization time of less than one week. 
Cardiovascular (39.9\%) and respiratory system (19.3\%) changes were the main reasons for hospitalization. Associated comorbidities were absent in $57.9 \%$ of patients. Regarding the hospitalization outcome, 55.45\% were discharged from the hospital, $36.45 \%$ died and $8.10 \%$ were transferred; $52 \%$ of patients required mechanical ventilation during hospitalization. Conclusion: This study recognized the profile of the public attended in an ICU of middleaged men, affected by cardiovascular events, serving as a subsidy for the adoption of health practices of prevention, promotion and rehabilitation specific to the identified public.

Keywords: Health Profile; Intensive Care Unit; Physiotherapy.

\section{INTRODUÇÃO}

A carência de leitos e profissionais habilitados, para atendimento em unidades de terapia intensiva e a racionalização das admissões devido aos elevados custos e disposição de recursos de alta tecnologia, tornou-se um problema de saúde pública devido ao aumento da demanda em relação à oferta. Esse quadro é denominado como crise do atendimento intensivista ${ }^{1}$.

A Unidade de Terapia Intensiva (UTI) é destinada para assistência de pacientes instáveis e graves. É classificada com um ambiente de alta complexidade devido aos aparelhos de alta tecnologia e equipe especializada de forma integral. É categorizada em nível 1, 2 e 3, de acordo a quantidade de serviços presentes².

$\mathrm{Na}$ equipe multidisciplinar, em terapia intensiva, o fisioterapeuta é responsável pelo atendimento de pacientes críticos, suporte ventilatório e assistência pré e pós-cirúrgica. A fisioterapia intensiva tem objetivos terapêuticos e profiláticos como aumentar a capacidade funcional geral, restaurar a independência, diminuição de complicações associadas ao repouso no leito e promoção bem estar do paciente ${ }^{3}$.

Devido a grande demanda nas UTI's e pouca disponibilidade de investimento, nesse âmbito, tornase necessário conhecer o perfil da clientela para adequado manejo da população além do planejamento e estruturação do sistema de saúde. Diante do exposto, essa pesquisa buscou identificar o perfil dos pacientes atendidos pela fisioterapia na Unidade de Terapia Intensiva do Hospital Regional de Eunápolis (HRE) durante o período entre março de 2019 a março de 2020.

\section{MÉTODO}

Foi realizado um estudo transversal, documental, retrospectivo, de caráter exploratório e abordagem descritiva, através da análise de prontuários de pacientes admitidos e atendidos pela fisioterapia na Unidade de Terapia Intensiva do Hospital Regional de Eunápolis - BA, no período de 01 de março de 2019 à 31 de março de 2020 .

Foram selecionados apenas os prontuários de pacientes maiores de 18 anos atendidos no período da pesquisa pela fisioterapia na UTI adulto e excluídos os prontuários em que o paciente ficou menos de 24 horas na UTI. As variáveis analisadas foram divididas em dois grupos: Características sociodemográficas (idade, sexo, raça, estado civil e escolaridade) e características clínicas (Motivo da internação, tempo de internação, data de admissão, alta hospitalar, transferência, óbito, doenças de base, comorbidades associadas, ventilação mecânica, tempo de ventilação mecânica, traqueostomia e intubação orotraqueal). Os dados foram armazenados em planilha eletrônica e analisados de maneira descritiva no Microsoft Office Excel (2013).

A coleta de dados foi respaldada e realizada respeitando os preceitos da Resolução 466/2012 do Conselho Nacional de Saúde, os dados foram coletados de forma secundária, via prontuário, disponibilizado pelo setor da UTI e todos os indivíduos envolvidos ou responsáveis assinaram o TCLE - Termo de consentimento livre e esclarecido. O estudo foi aprovado pelo Comitê de Ética e Pesquisa da Universidade Estadual de Santa Cruz - UESC, com CAEE 30003520.5.0000.5526 e parecer $n^{\circ} 3.975 .088$.

\section{RESULTADOS}

Durante o período de coletas, 321 prontuários atenderam aos critérios de inclusão e foram analisados.

Houve predominância de pacientes do sexo masculino representando $54 \%$ das admissões, com média de idade de 58,64 anos e desvio padrão de $\pm 18,9$. As variáveis escolaridade, estado civil, raça/cor não apresentam validade estatística, nessa pesquisa, devido ao missing de dados, ou seja, não foi possível a coleta por não estar presente no prontuário, como pode ser visto na Tabela 1.

Através da Figura 1 é possível conhecer os principais motivos de internação com predominância dos eventos cardiovasculares seguido das patologias respiratórias. Quanto as comorbidades associadas, 57,9\% ( $n=186)$ dos pacientes não apresentaram nenhum tipo de comorbidades, 32,1\% ( $n=103)$ com 1 comorbidade, 8,4\% $(n=27)$ com 2 comorbidades e apenas $1,6 \%(n=5)$ com 3 ou mais comorbidades associadas.

A Tabela 2 traz a distribuição dos pacientes que necessitaram de Ventilação Mecânica invasiva e não invasiva. Verificou-se que o tempo de internação teve uma média de 8 dias com desvio padrão de $\pm 18,91$, a quantidade de semanas, bem como, o desfecho da internação estão representados na Tabela 3.

O período com maior número de admissões foi em março de 2019, com um total de 36 indivíduos 
Tabela 1. Perfil sociodemográfico dos pacientes atendidos na Unidade de Terapia Intensiva adulto no período de 01 de março de 2019 a 31 de março de 2020.

\begin{tabular}{|c|c|c|}
\hline Variáveis & $\mathrm{N}^{\circ}$ & $\%$ \\
\hline \multicolumn{3}{|l|}{ Sexo } \\
\hline Masculino & 174 & 54 \\
\hline Feminino & 147 & 46 \\
\hline \multicolumn{3}{|l|}{ Faixa etária (anos) } \\
\hline 18 a 19 - Jovens & 4 & 1 \\
\hline 20 a 59 - Adultos & 149 & 46 \\
\hline$>60-$ Idosos & 168 & 52 \\
\hline \multicolumn{3}{|l|}{ Raça/cor } \\
\hline Preta & 10 & 3 \\
\hline Pardo & 103 & 32 \\
\hline Branca & 9 & 3 \\
\hline Sem informação & 199 & 62 \\
\hline \multicolumn{3}{|l|}{ Escolaridade } \\
\hline Analfabeto & 36 & 11 \\
\hline Fundamental Incompleto & 57 & 18 \\
\hline Fundamental Completo & 5 & 2 \\
\hline Ensino Médio Incompleto & 18 & 6 \\
\hline Ensino médio Completo & 1 & 0 \\
\hline Ensino Superior Incompleto & 1 & 0 \\
\hline Ensino Superior Completo & 2 & 1 \\
\hline Não Informado & 201 & 63 \\
\hline \multicolumn{3}{|l|}{ Estado Civil } \\
\hline Casado & 34 & 11 \\
\hline Solteiro & 35 & 11 \\
\hline Divorciado & 5 & 2 \\
\hline União estável & 33 & 10 \\
\hline Viúvo & 16 & 5 \\
\hline Não Informado & 198 & 62 \\
\hline
\end{tabular}

Resultados expressos através de análises de frequência.

representando $11,2 \%$ do total de internações, no período da pesquisa, como representado na Figura 2.

\section{DISCUSSÃO}

O presente estudo demonstrou uma prevalência na admissão indivíduos do sexo masculino, representando $54 \%(n=174)$ do total da amostra, corroborando com o estudo de França et al. ${ }^{4}$ que realizaram um estudo de perfil epidemiológico da unidade de terapia intensiva de um hospital universitário, envolvendo 102 internos
Tabela 2. Distribuição dos pacientes que necessitaram de Ventilação Mecânica Invasiva e Ventilação Não Invasiva na Unidade de Terapia Intensiva adulto entre 01 de março de 2019 a 31 de março de 2020.

\begin{tabular}{lcc}
\multicolumn{1}{c}{ Variáveis } & $\mathrm{N}^{\circ}$ & $\%$ \\
\hline Ventilação Mecânica & 167 & 52 \\
Sim & 154 & 48 \\
Não & 152 & 91 \\
VMI & 140 & 91 \\
Intubação Orotraqueal & 12 & 8 \\
Traqueostomia & 15 & 9 \\
VNI &
\end{tabular}

Resultados expressos através de análises de frequência. VMI: Ventilação Mecânica Invasiva; VNI: Ventilação Não Invasiva.

Tabela 3. Distribuição dos pacientes segundo tempo e desfecho da internação na Unidade de Terapia Intensiva adulto entre 01 de março de 2019 a 31 de março de 2020.

\begin{tabular}{lcc}
\hline \multicolumn{1}{c}{ Variáveis } & $N^{\circ}$ & $\%$ \\
\hline Tempo de internação & & \\
<1 semana & 125 & 39 \\
1-2 Semanas & 121 & 38 \\
2-3 Semanas & 58 & 18 \\
3-4 Semanas & 9 & 3 \\
> 1 mês & 8 & 2 \\
Desfecho da internação & & \\
Alta Hospitalar & 178 & 55 \\
Transferência & 26 & 8 \\
Óbito & 117 & 36 \\
\hline
\end{tabular}

Resultados expressos através de análises de frequência.

na unidade, onde $55,9 \%(n=57)$ das admissões eram do sexo masculino. Se assemelhando também aos estudos de Brito $^{5}$ onde pesquisou o perfil epidemiológico dos pacientes internos na UTI do Hospital Universitário Onofre Lopes, no Rio Grande do norte, com uma um total de 1095 pacientes para realização da pesquisa e verificou que $55,35 \%(n=606)$ das internações eram de pacientes do sexo masculino.

Diversos estudos possuem resultado semelhante e pode ser justificado pela baixa adesão masculina aos cuidados à saúde e maior exposição deste público à atividades/condutas de risco. Normalmente, quando acontece a adesão masculina aos serviços de saúde, muitas vezes, o nível de gravidade da patologia já está avançado devido à procura tardia por serviços de saúde. Como demonstrado no estudo de Levorato e colaboradoes ${ }^{6}$ sobre os fatores associados à procura por 


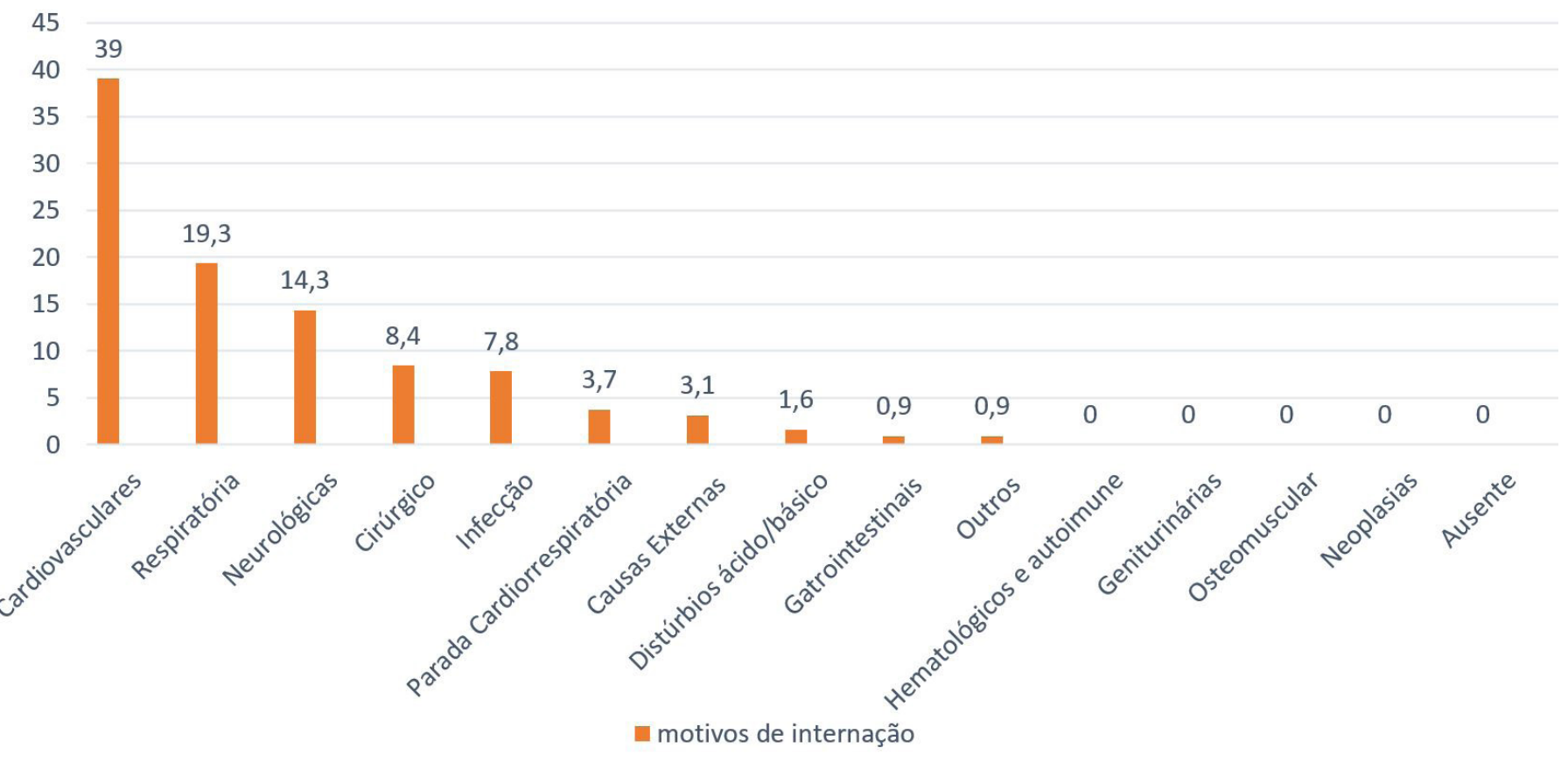

Figura 1. Principais motivos de internação na Unidade de Terapia Intensiva adulto no período de 01 de março de 2019 a 31 de março de 2020. Resultados expressos através de análises de frequência.

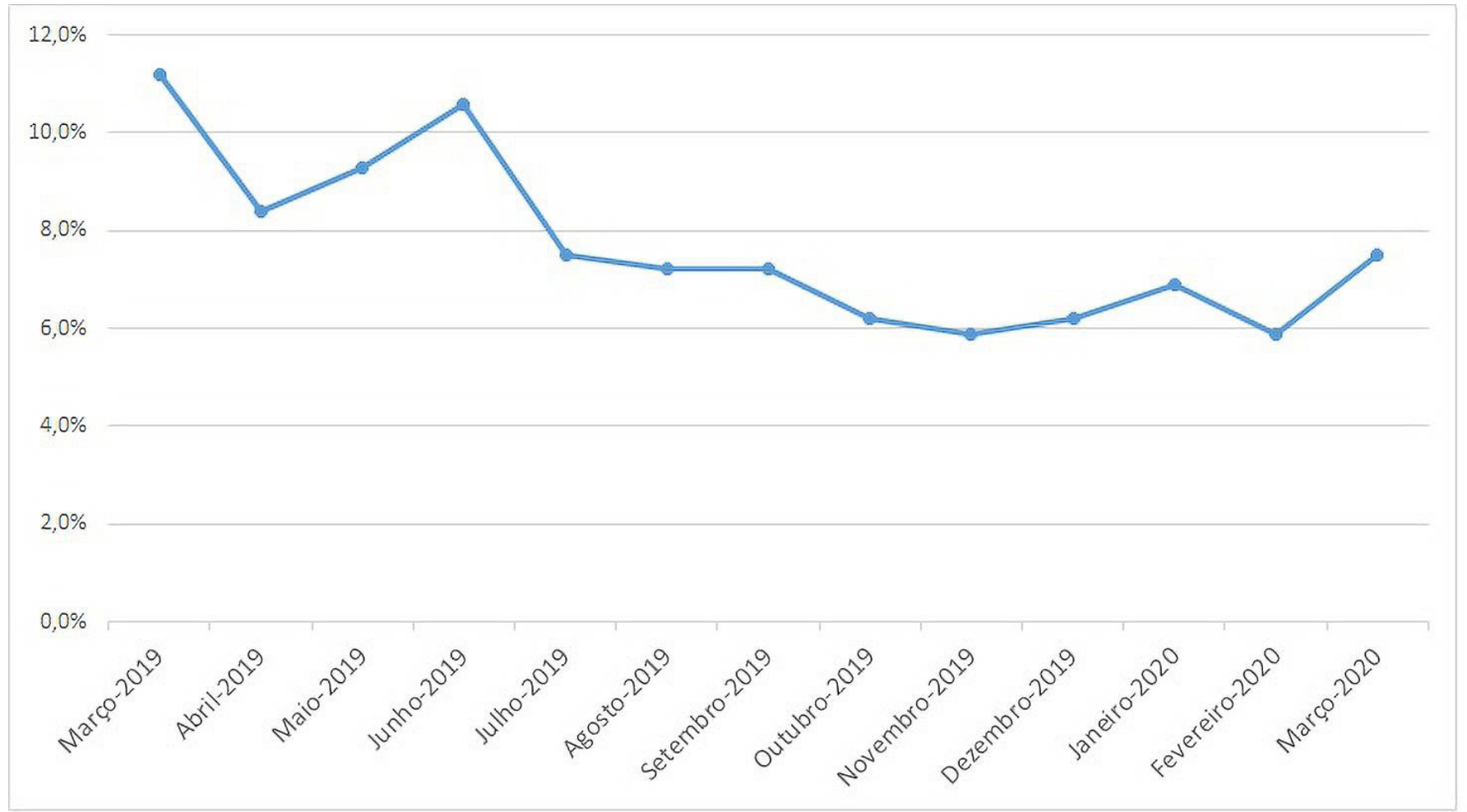

Figura 2. Acompanhamento das admissões na Unidade de Terapia Intensiva adulto entre 01 de março de 2019 a 31 de março de 2020. Resultados expressos através de análises de frequência.

serviços de saúde numa perspectiva relacional de gênero, onde se observou a prevalência das mulheres na busca por serviços de saúde, representando 1,9 vezes mais em relação aos homens, onde ser do sexo feminino foi um fator preditor de maior busca por assistência à saúde, sendo mensurado com magnitude de 2,43 vezes em relação ao sexo masculino.
A faixa etária com maior porcentagem de internação foi $>60$ anos - idosos representando 52\% $(n=168)$ da amostra, assim como no estudo de Guia et al. ${ }^{7}$, que realizaram um estudo na UTI de um hospital público no Distrito Federal com um total de 189 pacientes, onde a maioria, 94,6\% ( $n=179)$ apresentava idade superior a 60 anos. Pauletti et al. ${ }^{8}$ demonstraram em seu estudo uma 
prevalência, em internamentos, da população acima de 60 anos representando 46,9\% (n=457) da amostra do estudo. Esse fenômeno é justificado pela transição demográfica e consequente alteração no perfil epidemiológico, fazendo com que as taxas de internação dos grupos idosos sejam mais elevadas, caracterizada pela maior prevalência de doenças crônico-degenerativas que estão diretamente relacionadas com a idade. O tratamento dessas doenças demanda a utilização de serviços de maior complexidade, com alto nível tecnológico.

Os principais motivos de internação encontrados nesta pesquisa foram àqueles relacionados às alterações do sistema cardiovascular correspondendo a 40\% $(n=128)$ da amostra, seguido por alterações respiratórias 19\% $(n=62)$. Semelhante ao resultado do estudo de Brito ${ }^{5}$ onde a maior causa de internação foi, também, por comprometimento do sistema cardiovascular totalizando 49\% $(n=647)$ da amostra da pesquisa, seguido pelas disfunções respiratórias com um total de $21 \%(n=236)$. Esses resultados se diferem do estudo de Carvalho e autores $^{9}$, onde os motivos de internação mais frequentes foram de origem neurológicas 32\% $(n=17)$ e traumática $25 \%(n=14)$. Essa variação dos motivos de internação se dá devido a diversos fatores, dentre eles podemos considerar que o Brasil possui uma heterogeneidade demográfica, social e econômica muito grande, que se reflete em diferentes padrões de patologias.

Em relação às comorbidades associadas, neste estudo, 58\% ( $n=186)$ dos pacientes não apresentaram e $42 \%(n=135)$ apresentaram uma ou mais comorbidades, mostrando certa semelhança com outros estudos como o de Bhering ${ }^{10}$ onde $95,02 \%$ ( $n=267$ ) dos pacientes do estudo não apresentaram comorbidades e 4,98\% ( $n=14)$ tinham uma ou mais comorbidades associadas.

Com a transição demográfica, o Brasil, sofreu alterações no perfil de doenças que afetam a população, saindo do quadro com surtos e epidemias de doenças infectocontagiosas para uma população que apresenta, em sua maioria, patologias crônicas não degenerativas, ou seja, as comorbidades associadas. Como as comorbidades associadas muitas vezes apresentam evolução silenciosa, a falta de diagnóstico pode ser um fator determinante no resultado da pesquisa, como encontrado nos estudos de Capilheira e Santos ${ }^{11}$, que apontou a precariedade na busca pelo diagnóstico precoce das doenças crônicas não degenerativas e comorbidades associadas. Tais dados devem chamar atenção dos diversos setores de gestão pública da saúde, a fim de fortalecerem os projetos, programas e discussões em torno do rastreamento e diagnóstico precoce das diversas doenças crônico degenerativas.

Ao investigar o desfecho da internação foi encontrado que $55 \%(n=178)$ dos pacientes admitidos tiveram alta hospitalar, 36\% ( $n=117)$ evoluíram para óbito e $8 \%(n=26)$ necessitaram de transferência. Bueno e Biatto ${ }^{12}$, ao estudarem a epidemiologia de uma UTI mista do norte do Paraná relataram que, em relação ao desfecho dos pacientes, $72,53 \%(n=169)$ tiveram alta e $27,47 \%(n=64)$ evoluíram para óbito, o resultado de alta hospitalar é superior ao que encontrado nesse estudo. Já no estudo de França, Albuquerque e Santos ${ }^{4}$ foi relatado que 47,1\% ( $n=48)$ obtiveram alta, 47,1\% ( $n=48)$ evoluíram para óbito e 5,8\% (n=6) necessitaram de transferência, mostrando uma taxa de óbito superior e uma taxa de alta hospitalar inferior ao presente estudo.

A média de dias que um paciente fica internado na UTI da instituição investigada é de aproximadamente 8 dias, com predominância da permanência inferior a 1 semana correspondendo a 39\% $(n=125)$ dos casos. Tais achados foram semelhantes aos encontrados por Sousa et al. ${ }^{13}$, onde em um estudo epidemiológico na UTI do Hospital Regional de Cajazeiras foi descrito que a maior parte das internações, 82,6\% ( $n=256)$ ficou entre 1 a 10 dias. Esses resultados trazem à tona o conceito primordial de UTI, uma unidade hospitalar com intervenções intensas que tem por objetivo estabilizar e recuperar o enfermo no menor tempo possível, evitando grandes períodos de internação que têm associação direta com a gravidade prognóstica, complicações, efeitos deletérios e aumento dos custos para os serviços de saúde.

No presente estudo foi possível observar que 52\% $(n=167)$ dos pacientes necessitaram de ventilação mecânica no decorrer da internação, similar ao estudo de Bueno e Biatto ${ }^{12}$ que relataram que a principal medida de suporte utilizada na UTI geral do Hospital Santa Rita de Maringá-PR, foi a ventilação mecânica 42,06\% ( $n=98)$. Sendo um procedimento de cuidados intensivos podese notar que os pacientes da Unidade realmente são pacientes críticos. A escolha por esta técnica justifica-se no fato do grau de eficiência da técnica em dar suporte ou substituir a ventilação espontânea, sendo um recuso de alta complexidade e tecnologia que o ambiente da UTI dispõe.

Com relação ao tipo de ventilação mecânica 91\% $(n=152)$ necessitaram de VMI e 9\% $(n=15)$ VNI, diferente do estudo de Lisboa et al. ${ }^{14}$ onde $64,81 \%(n=105)$ usaram VNI e somente $35,19 \%$ ( $n=57)$ usaram VMI. A escolha de uma determinada forma e modalidade de ventilação depende muito da expertise da equipe. Ao comparar os resultados da utilização de VMI e VMNI, podemos ver que os pacientes utilizaram mais a VMI, por serem, em grande parte, pacientes com doenças cardiovasculares e neurológicas que, em seu quadro clinico, podem apresentar instabilidade hemodinâmica e rebaixamento do nível de consciência, dois critérios de exclusão para uso de VMNI. Além disso, a demora pela liberação da vaga de UTI pode contribuir para que estes pacientes sejam admitidos com maior gravidade do quadro, com a VMNI não mais fazendo o efeito desejado.

Apesar de ter sido um estudo puramente descritivo, o perfil aqui traçado reforça a necessidade de reestruturação das estratégias de prevenção e promoção à saúde, para 
o controle dos eventos cardiovasculares por causas evitáveis. Fica evidente que cada região e cada grupo populacional possuem suas particularidades, ressaltando a importância de estudos epidemiológicos regionais para melhor preparação das unidades, equipes, melhor manejo e assistência voltados às necessidades da demanda local.

\section{CONCLUSÃO}

A partir da análise de 321 prontuários de pacientes admitidos e atendidos pela fisioterapia na Unidade de Terapia Intensiva do Hospital Regional de EunápolisBahia, no período de março de 2019 à março de 2020, foi conhecido o perfil predominante de paciente do sexo masculino, com média de 58 anos, internado por evento cardiovascular que em menos de uma semana evoluiu para ventilação mecânica e desfecho positivo de alta hospitalar.

\section{FONTE DE FINANCIAMENTO}

Nada a declarar.

\section{CONFLITO DE INTERESSES}

Nada a declarar.

\section{REFERÊNCIAS}

1. Medeiros R. Insuficiência de leitos de UTI: crise do capital e mercantilização da saúde. Argumentum. 2018 Jun 28;10(1):229-40. http://dx.doi.org/10.18315/argumentum. v10i1.18647.

2. Backes MT, Erdmann AL, Büscher A. The living, dynamic and complex environment care in intensive care unit. Rev Lat Am Enfermagem. 2015 Jul 3;23(3):411-8. http://dx.doi. org/10.1590/0104-1169.0568.2570. PMid:26155009.

3. Nunes Alves A. A importância da atuação do fisioterapeuta no ambiente hospitalar. Ensaios e Cienc: Cienc Biol Agrar Saude 2014;16(6):173-84.

4. França CDM, Albuquerque PR, Santos ACBC. Perfil epidemiológico da unidade de terapia intensiva de um hospital universitário. InterScientia. [Internet]. 2013 [citado em 2020 Out 29];1(2):72-82. Disponível em: https:// periodicos.unipe.br/index.php/interscientia/article/view/37

5. Brito JRF. Perfil epidemiológico dos pacientes internos na unidade de terapia intensiva do hospital universitário Onofre Lopes [monografia]. Rio de Janeiro: Universidade Federal do Rio de Janeiro; 2017.

6. Levorato CD, Mello LM, Silva AS, Nunes AA. Fatores associados à procura por serviços de saúde numa perspectiva relacional de gênero. Cien Saude Colet. 2014;19(4):1263-74. http:// dx.doi.org/10.1590/1413-81232014194.01242013.

7. Guia CM, Biondi RS, Sotero S, Lima AA, Almeida KJQ, Amorim FF. Perfil epidemiológico e preditores de mortalidade de uma Unidade de Terapia Intensiva geral de hospital público do Distrito Federal. Com Ciênc Saúde. 2015;26(1-2):9-19. https://doi.org/10.51723/ccs.v26i01/02.156.

8. Pauletti M, Otaviano MLPO, Moraes AST, Schneider DS. Perfil epidemiológico dos pacientes internados em um Centro de Terapia Intensiva. Aletheia. [Internet]. 2017 [citado em 2020 Out 29];50(1-2):38-46. Disponível em: http://pepsic. bv salud.org /scielo.php?script=sci_arttext\&pid=S141303942017000100004\&lng=pt

9. Carvalho N, Silva M, Paula P, Pires J, Yamaguchi M, Costa C. Principais causas de internamento na unidade de terapia intensiva em um hospital de Maringá - PR. In: Anais Eletrônicos da VI Mostra Interna de Trabalhos de Iniciação Cientifica [Internet]. Maringá: Unicesumar; 2013 [citado em 2020 Out 29]. Disponível em: http://www.cesumar.br/ prppge/pesquisa/epcc2013/oit_mostra/Naiara_Zanquetta_ Carvalho.pdf

10. Bhering CFC. Fatores associados à readmissão em uma Terapia Intensiva [dissertação]. Rio de Janeiro: Universidade Federal do Estado Rio de Janeiro; 2016.

11. Capilheira M, Santos IS. Doenças crônicas não transmissíveis: desempenho no cuidado médico em atenção primária à saúde no sul do Brasil. Cad Saude Publica. 2011;27(6):114353. http://dx.doi.org/10.1590/S0102-311X2011000600011.

12. Bueno $\mathrm{H}$, Biatto J. Epidemiologia e validação de escore prognóstico em UTI mista do Norte do Paraná. UNINGUÁ Rev. [Internet]. 2015 [citado em 2020 Out 29];22(3):2329. Disponível em: http://revista.uninga.br/index.php/ uningareviews/article/view/16 32

13. Sousa MNA, Cavalcante AM, Sobreira REF, Bezerra ALD, Assis EV, Feitosa ANA. Epidemiologia das internações em uma unidade de terapia intensiva. Rev Eletrônica FAINOR, 2014;7(2):178-86.

14. Lisboa DJ, Medeiros EF, Alegretti LG, Badalotto D, Maraschin R. Perfil de pacientes em ventilação mecânica invasiva em uma unidade de terapia invasiva. J Biotechnol Biodivers. 2012;3(1):18-24. 